\title{
Examination of Time-Varying Kinematic Responses to Support Surface Disturbances
}

\author{
S. Gurses", R.V. Kenyon ${ }^{* *}$, E. A. Keshner \\ ${ }^{*}$ Middle East Technical University, Dept. of Engineering Sciences, Ankara 06531, \\ Turkey (Tel: +90-312-210 4461; e-mail:senih@metu.edu.tr). \\ ${ }^{* *}$ University of Illinois at Chicago, Dept. of Computer Science, Chicago, IL 60607 \\ USA(e-mail:kenyon@uic.edu). \\ ${ }^{* * *}$ Dept. of Physical Therapy and Dept. of Electrical and Computer Engineering, Temple University, \\ Philadelphia PA 19095 USA (e-mail: ekeshner@temple.edu).
}

\begin{abstract}
To examine the evolution of inter-segmental coordination over time, a previously developed multi-variate model of postural coordination during quiet stance (Kuo et al. 1998) has been extended. In the original model, postural coordination was treated as an eigenvalue-eigenvector problem between two segmental degrees of freedom represented by angular displacements of the trunk and lower limb. Strategies of postural coordination were then identified using the sign of the covariance between the two segments' angular displacements. In contrast to the original model, the current model first subdivided the entire trial into smaller time segments, comprising four cycles of perturbation, i.e., a $16 \mathrm{sec}$ window. This window marched along the data advancing in $8.3 \mathrm{~ms}$ steps, each time performing the computation from the original model on the terms of the covariance matrix. The resulting time-segment-dependent postural strategies estimated the changes in posture control that took place over the course of the experiment. In addition to the statistical modeling, the auto-power spectrums and cross-spectral density function estimates for the entire trial, as well as for the individual time-segments, were analyzed. In these experiments subjects experienced a $0.25 \mathrm{~Hz}$ sinusoidal perturbation of a platform while exposed to a virtual reality environment. The data we collected showed that the statistical and spectral characteristics across the entire trial may differ from individual time segments of the same trial indicating time varying postural behavior. Comparison of these results from young and elderly subjects revealed that the time dependency observed in postural behavior was sensitive to aging. The young population managed to be consistent in their postural behavior throughout the entire trial and responded to the perturbation frequency with an out-of-phase response between the postural segments. Elderly subjects, however, demonstrated inconsistent postural behaviors as they switched back and forth between different postural coordination patterns within a trial.
\end{abstract}

Keywords: posture; statistical model; postural strategy; spectral content; time dependency.

\section{INTRODUCTION}

Previous studies have shown that sway during quiet stance (Carroll and Freedman 1993; Collins and De Luca 1993; Schumann et al. 1995; Loughlin et al. 1996; Creath et al. 2005; Guelton et al. 2008) exhibits non-linear and timevarying behavior suggesting shifting mechanisms for control. Furthermore, Creath et al. (2005) have proposed that the whole body behaves like a multi-link pendulum during quiet stance and has two simultaneously co-existing excitable modes. Each mode exhibits a separate eigen-frequency containing characteristic phase relationships that are functions of the frequency at which the individual segments oscillate. Time-varying spectral characteristics in head and trunk stabilization to an external perturbation (Gurses et al. 2005) have also recently been reported, implying that segmental adaptation to an external disturbance may continuously shift between several control mechanisms. Kuo et al. 1998 developed a multi-variate model of postural coordination during quiet stance. In their model, postural coordination was treated as an eigenvalue-eigenvector problem between two segmental degrees of freedom represented by angular displacements of the trunk and lower limb. A multivariate description of postural sway was constructed to reveal two independent kinematic (spatial) relationships (hip and ankle eigen-movements) each defining a postural strategy (Kuo et al 1998). A hip or ankle strategy of postural coordination was identified using the sign of the covariance between the two segments angular displacements.

To understand the non-linear and time-varying properties involved in posture control we extended the Kuo et al (1998) model by dividing a trial into several time segments and then analyzing these time segments to identify hip or ankle strategies. The ensuing data provided us with a picture of the evolution of inter-segmental coordination across a trial. Our results from examining both young and elderly populations showed that a majority of the young subjects exhibited a consistent postural control strategy across time, whereas the elderly subjects were more likely to change their postural control strategy from one time segment to the next. Moreover, the characteristics of the spectral components were found to be stable across time in the young subjects, 
pointing to a consistent postural strategy, that is, an out of phase relationship at the perturbation frequency. On the other hand, elderly subjects demonstrated large variability in their postural responses such that many frequencies were found to be involved in their postural coordination.

\section{METHODS}

\subsection{Previous Model}

Eigenvector / Eigenvalue Structure of the Problem through a Statistical Model: Kuo et al. 1998 used a multivariate description of postural sway to reveal two independent kinematic (spatial) relationships (eigen-movements) each defined as a postural strategy: an inverted pendulum (ankle strategy) or a bending at the hip (hip strategy) (Horak and Nashner 1986). In this model, the output vector $\theta$ for the kinematic relationship between two segments was defined by 1 , where $\theta_{\mathrm{a}}$ and $\theta_{\mathrm{h}}$ represent the angle between the foot and the shank (ankle) and the angle between the leg and the trunk (hip), respectively. Variability between the motions of these two segments was calculated using the covariance matrix Q (2) where $n$ is the number of data points. Matrix $Q$ is a symmetric matrix, where co-variance of ankle and hip $\left(\sigma_{\mathrm{ah}}\right)$ is equal to the covariance of hip and ankle $\left(\sigma_{\mathrm{ha}}\right)$. Further, $\sigma_{\mathrm{a}}{ }^{2}$ and $\sigma_{\mathrm{h}}{ }^{2}$ in (2) are variance of angular motion at ankle and hip respectively. Eigenvalues and related eigenvectors of this covariance matrix were computed to extract the spatial relation between the angular displacement of the trunk and the shank. Eigenvector 1 was defined as representing an "inverted pendulum" strategy, where both segments rotate in the same direction. Similarly, eigenvector 2 was defined representing the "hip strategy" where the two segments rotate in opposite directions. The amplitudes of the related segmental motions defined the associated eigenvalue of the eigenvectors since this scaled the length of the vectors and did not affect their direction (Fig. 1e).

$$
\begin{aligned}
& \theta=\left[\begin{array}{ll}
\theta_{a} & \theta_{h}
\end{array}\right] \\
& Q=\frac{1}{n-1} \sum_{i=1}^{n}\left(\theta_{i}-\bar{\theta}\right)^{T}\left(\theta_{i}-\bar{\theta}\right)=\left[\begin{array}{cc}
\sigma_{a}^{2} & \sigma_{a h} \\
\sigma_{h a} & \sigma_{h}^{2}
\end{array}\right]
\end{aligned}
$$

Geometric Representation of the Solution: Kuo et al. (1998) used a two degree of freedom (DOF) statistical multivariate model to analyze postural sway. Plotting the angular motion of the shank vs. the trunk results in an ellipse which lay in a plane labeled P (Fig. 1). The ellipse graphically demonstrated the dominant postural strategy used by the subject throughout the selected interval of time. The major and minor axes of the ellipse in the plane $\mathrm{P}$ represented two spatial relationships (eigenvectors) between the angular motion of the shank and the trunk. The covariance matrix $Q$ was decomposed (diagonalization) to identify these spatial relationships inherent in the ellipse. The angle of the ellipse's major axis $(\alpha)$ designated the eigenvector associated with the largest eigenvalue of the co-variance matrix, Q. A counterclockwise rotation of the angle $\alpha$ (from the $+\mathrm{x}$-axis) was defined as positive. The dominant postural strategy used by the subject was deemed an ankle strategy if $\pi / 2<\alpha<0$ (quadrant I response), or a hip strategy if $\pi<\alpha<\pi / 2$ (quadrant II response).

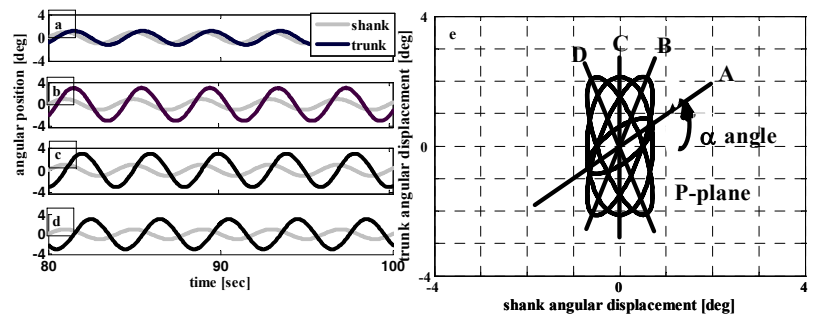

Fig. 1. a) A $20 \mathrm{sec}$ portion of a $140 \mathrm{sec}$ time series simulation of trunk and shank angular position data during a $0.25 \mathrm{~Hz}$ platform translation. $a$ and b) Amplitude of the trunk's motion decreased by a factor of 3 with respect to the angular motion of the shank but the phase angle in between remained constant $(\varphi=\pi / 4)$. c and d) Amplitude of the trunk's angular motion with respect to the angular motion of the shank remained constant but $\varphi$ shifted ( $\pi / 2$ and $3 \pi / 4$, respectively). e) Elliptical representation of the data simulated in $1 \mathrm{a}, \mathrm{b}, \mathrm{c}$, and $d$ on the P-plane.

\subsection{Time Domain Statistical Model}

Time Domain Analysis: Kuo et al. (1998) used the subject's entire response during a trial to characterize their behavior. In this study, however, we investigated how postural characteristics varied during the period of a trial by subdividing each trial into time segments. By analyzing each time segment individually we could examine the variability of the postural coordination exhibited by the subject during the trial. Consequently, the terms of the covariance matrix $Q$ in (2) became a function of the $\mathrm{m}$ time segments that comprised the experimental data. That is, the covariance term $\sigma_{\text {ah }}$ in (2) is applied to a single time segment where $T$ covers the time interval for a single time segment in the (3).

$$
\sigma_{a h}=\frac{1}{T} \int_{0}^{T}\left\{\theta_{a}(t)-\bar{\theta}_{a}\right\}\left\{\theta_{h}(t)-\bar{\theta}_{h}\right\} d t
$$

In essence, (3) characterizes the postural behavior for a specific time segment. When this analysis is performed for each time segment that is part of the entire trial, $\sigma_{a h}$ becomes a function of time.

Since only the sign of the covariance can indicate a change in the postural strategy, the covariance was normalized with respect to the magnitude of the motion of each of the body segments. This normalization transformed the covariance into the correlation coefficient thereby making its sign the prevalent variable (eq. 4,5; Bendat and Piersol 1993). As a result, the sign of the covariance of the motion between shank and trunk was used to extract the dominant eigenvector associated with the largest eigenvalue.

$$
\rho_{a h}=\frac{\sigma_{a h}}{\sqrt{\sigma_{a a} \cdot \sigma_{h h}}}
$$

where

$$
-1 \leq \rho_{a h} \leq 1
$$




\subsection{Frequency Domain Analysis}

In addition to the statistical analysis above, power spectral density (PSD) function estimates and cross-spectral density (CSD) function estimates of the shank and trunk's motion for both the entire period of trial and for the individual time segments were also analyzed. The aggregate PSD and CSD for each time segment throughout the data will reveal any time-dependency effects that may exist in eigen-movements and related eigen-frequencies of the postural coordination. CSD estimates of the shank versus the trunk were then computed for each of the time segments to explore any time dependency that might be exhibited in the phase relationship between the shank and the trunk. Finally, the coherence function estimates between the shank and trunk's motion were computed to examine how the motions of the two body segments were coupled at the driving frequency.

\subsection{Experimental Apparatus and the Experimental Protocol}

Our model was applied to previously collected data from 7 healthy young (25-38 yrs) and 6 (60-78 yrs) healthy elderly subjects standing on a platform that translated in the anteriorposterior direction at $0.25 \mathrm{~Hz}$ for $180 \mathrm{sec}$ for the young and $90 \mathrm{sec}$ for the elderly. All subjects gave informed consent according to the guidelines of the Institutional Review Board of Feinberg School of Medicine, Northwestern University to participate in this study. All subjects had no history of central or peripheral neurological disorders or problems related to movements of the spinal column (e.g., significant arthritis or musculoskeletal abnormalities) and a minimum of 20/40 corrected vision.

Three-dimensional kinematic data from the body segments were collected at $120 \mathrm{~Hz}$ using a motion analysis system (Optotrak, Northern Digital Inc., Ontario, Canada) with a resolution of $0.1 \mathrm{~mm}$. Infrared markers placed at $\mathrm{C}_{7}$ and the greater trochanter were used to calculate trunk angular position relative to earth vertical. Shank angular position was defined as the angle between the lateral femoral condyle and the lateral malleolus relative to earth vertical. Sled motion was subtracted from the linear motion of each segment prior to calculating segmental motion. Motion of the shank was removed from motion of the trunk to reveal motion of the trunk with respect to the shank $\left(\theta_{h}\right)$. The shank was examined with respect to the platform $\left(\theta_{a}\right)$.

Subjects stood in front of a virtual environment that provided motion of the visual field. The virtual environment scene was driven by an external signal summed with movements of the subject's head transmitted from the motion analysis system (Keshner and Kenyon 2004). The time series for the shank and trunk angular displacements were de-trended linearly and bandpass filtered with a cut-off frequency of 0.05 and $50 \mathrm{~Hz}$. The first and last $20 \mathrm{sec}$ of each trial were removed leaving $140 \mathrm{sec}$ of the $180 \mathrm{sec}$ trials and $70 \mathrm{sec}$ of the $90 \mathrm{sec}$ trials to be analyzed. A $16 \mathrm{sec}$ window that marched along the data advanced $8.3 \mathrm{~ms}$ per step (1/120) to explore the time variations for each data sample.

\section{RESULTS}

Figure 2 shows a representative example of the behavior exhibited by the majority $(86 \%)$ of the young population and a minority of the elderly population (17\%) when experiencing a $0.25 \mathrm{~Hz}$ platform perturbation with an earthfixed scene. The single ellipse formed by using the entire response from this condition (Fig. 2a) and the multiple ellipses that resulted when these same data were subdivided into time segments (Fig. 2e) show similar characteristics. The pattern of postural behavior emerging from each method of analysis shows a well defined ellipse whose major axis lies in quadrant II of the P plane [R-square is 0.78 ]. The regularity of this response can be seen in Fig. $2 \mathrm{~d}$ where $\sigma_{a h}$, the covariance term, is plotted as a function of time. It has been found that $\sigma_{a h}$ has a consistent negative value throughout the epoch of the trial. The negative value of $\sigma_{a h}$ confirms that the angle $\alpha$, formed by the major axis of the ellipse, is in the second quadrant of the plane $\mathrm{P}$ for both the overall and the segmented ellipses (Fig. 2a\&e). A minor axis of the ellipse also emerges from the diagonalization of the covariance matrix $\mathrm{Q}$, and since it is orthogonal to $\alpha$, it is in the first quadrant for these same data.

To reveal the frequency components of the postural strategies used, we examined the power spectral density (PSD) of the two segmental motions over the entire trial and at each time segment. The PSD across the entire trial (Fig. 2b) shows that almost all of the power in this response occurs at the perturbation frequency $(0.25 \mathrm{~Hz})$. The PSD as a function of the individual time segments (Fig. 2f) shows approximately the same characteristic as does the entire trial analysis. However, the peak power varies somewhat over the course of the trial as seen in the middle of the time segment data. The CSD for the entire trial (Fig. 2c) reveals that these two body segments have a predominant out of phase $(180 \mathrm{deg})(\Phi$ : phase angle; $-\pi<\Phi<-\pi / 2$ or $\pi / 2<\Phi<\pi$ ) relationship at the perturbation frequency. When the trial was analyzed as a function of time (Fig. 2g), we found little difference in the phase at the disturbance frequency when compared to that of the entire trial. Furthermore, we find that the motion of the hip was larger than that of the shank. The two body segments are coupled and their motion is coherent with the perturbation at the $0.25 \mathrm{~Hz}$ frequency as shown in the coherence function of the two segments' motion (Fig. 2h). The power for the inphase relation of the two body segments (minor axis of the ellipse or ankle strategy) is small in the PSDs.

In contrast, a majority ( $83 \%$ ) of the elderly subjects and a minority of the young subjects (14\%) exhibited an overall ellipse that was not representative of the ellipses that emerged as a function of time. The single ellipse fitted using the entire trial of a representative elderly subject (Fig. 3a) falls in the second quadrant with an R-square of 0.43 . When these data were examined as a function of time, the individual ellipses that emerged changed in orientation, size, and shape (Fig. $3 e$ ). Thus, according to these data, unlike the majority of young subjects, a majority of the elderly do not exhibit a consistent sign for the covariance term (Fig. 3d). The value of $\sigma_{a h}(t)$ varied between \pm 1 across the time of the trial. These data quantify the extent to which $\alpha$ shifted between quadrants I and II. 


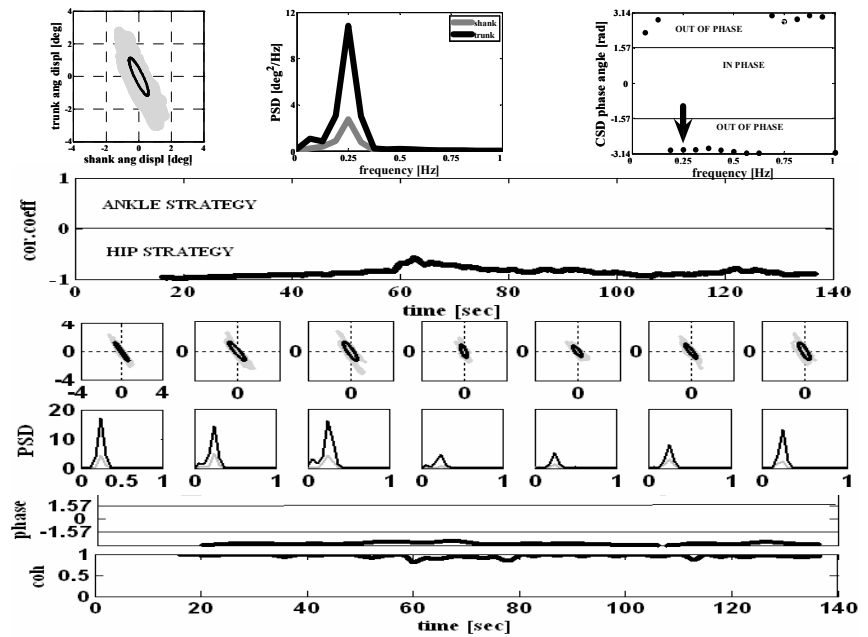

Fig. 2. a) The elliptical fit (black) to a 140 -sec response (grey) from a typical young subject plotted shank vs. trunk. All subsequent analyses used these data. b) PSD estimates for the entire trial: shank (grey) and trunk (black). c) CSD estimates for the entire trial (only phase angle shown). [Arrow indicates $0.25 \mathrm{~Hz}$ ]. d) Correlation coefficient, $\rho_{\mathrm{ah}}(\mathrm{t})$, between shank and trunk. e) Shank vs. trunk ellipses for seven different time segments. f) PSD at each time segment: shank (grey) and trunk (black). g) CSD at each time segment, $(\varphi$ at $0.25 \mathrm{~Hz}$.). h) The coherence function estimate at each time segment

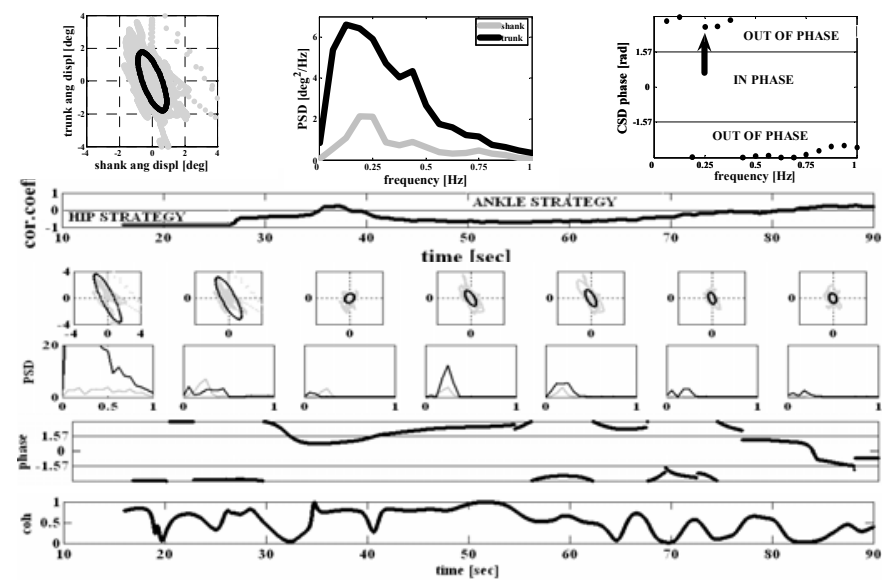

Fig. 3. a) 90-sec long angular position data of the shank with respect to platform (x-axis) plotted against angular position data of the trunk with respect to shank (y-axis) from a typical elderly subject. b, c, d, e, f, g, and h are structured identical to Fig. 2.

Examining the ensemble PSD produced for the shank and trunk using data from the entire epoch of the trial, we found that the power at the trunk was greater than that at the shank for all frequencies (Fig. 3b). Furthermore, the frequency at which the main peak occurred (Trunk: $0.13 \mathrm{~Hz}$; Shank: 0.2 $\mathrm{Hz})$ was different from the driving frequency $(0.25 \mathrm{~Hz})$. Furthermore, additional power can be seen out to $0.75 \mathrm{~Hz}$ for the trunk and $0.5 \mathrm{~Hz}$ for the shank. From the ensemble CSD phase (Fig. 3c), we found an out-of-phase relationship between the trunk and shank at all frequencies. The timesegmented PSDs (Fig. 3f) showed a more complex frequency relationship with time. The subject's postural responses exhibited peak power at multiple frequencies, instead of a single peak at the perturbation frequency. In addition, the dominant power shifted back and forth between shank and trunk as a function of time. Furthermore, the phase at the disturbance frequency $(0.25 \mathrm{~Hz})$ shifted from in-phase to outof-phase as a function of time (Fig. $3 \mathrm{~g}$ ). In $10 \%$ of this subject's time segments, the peak power was at the shank and the motion was in-phase (Fig. 3g); for the remaining 90\%, the peak power was at the trunk and the motion was generally out-of-phase. Accordingly, motion between the trunk and shank or between the body and the platform was rarely coherent (Fig. 3h).

\section{DISCUSSION}

Our analysis shows that the majority of the young adult subjects used a "hip strategy" when compensating for the postural disturbance. The use of the "hip strategy" at a single frequency meant that they only needed to control the phase of the segmental response to maintain a uniform postural coordination. Variability in the postural kinematics of an otherwise homogenous cohort (healthy young adults) has been noted previously when the sensory array has been significantly altered (Riley and Clark 2003) or when subjects were asked to perform a concurrent cognitive task (Keshner et al. 1995; Rankin et al. 2000; Keshner 2003). But when neither sensory alterations nor additional attention demands were present, healthy young subjects exhibited consistent ground reaction forces and lower limb EMG responses after the first few seconds of a $30 \mathrm{sec}$ trial of tandem stance (Jonsson et al. 2005). However, given the same single frequency stimulus perturbation, elderly adults exhibited multiple dominant frequencies in their response. The appearance of multiple frequencies required that they control phase, magnitude, and the frequency of the response to organize an effective postural strategy. In fact, elderly subjects were observed to switch their response strategies between hip and ankle through a continuum of these synergies throughout the period of the trial (Speers et al. 2002; Horak 2006).

During quiet stance, Creath et al. (2005) identified postural behaviors that had co-existing excitable modes, each with separate eigen-frequencies in young adults. Our analysis indicates that when a single dominant input perturbs the system, a more consistent strategy is organized by the young adults to counteract the disturbance with almost the entire power of the response observed at the perturbation frequency. Thus, postural control in young adults can demonstrate linear system behavior. On the contrary, our results indicate that the elderly subjects are unable to harness their control mechanisms to match the most relevant frequency for the postural disturbance. Rather, the power of the elderly subjects' response is scattered over a broad, nearly featureless spectrum. This spectrum resembles a chaotic regime developed by transitions originating from nonlinearities in the postural dynamics which have started from a few independent oscillations at well defined frequencies (Baker and Gollub, 1990). In fact, responses observed over long periods of postural disturbance would suggest that higher order processes (i.e., attention and perception) are supplying the non-linear dynamics to the postural behaviors recorded in 
these experiments (Nashner 1982; Shumway-Cook and Woollacott 1999; Redfern 2001; Buchanan and Horak 2001, 2003; Bottaro et al. 2005). The subsequent demands of controlling multiple response parameters will increase the complexity of the postural solution and may ultimately require that greater attention be given to the organization of the response. Diminished capacity to attend to more than one task during postural demands has been cited as a causative factor for instability in the elderly (Woollacott and Shumway-Cook 2002). Thus, these findings have significant implications for the control of posture in the elderly, particularly when placed in environments that contain more than one input that may have an impact on our ability to maintain an upright posture (Keshner and Kenyon, 2000; Keshner et al. 2004).

Any possible existence of chaotic behavior or motion, and the potential physiological mechanisms responsible for the behavioral difference observed between young and elderly subjects, deserves to be analyzed. We have demonstrated that a time domain statistical model provides a more sensitive measure of postural organization than the more commonly used measure of center of pressure (Jonsson et al. 2004, 2005; Barbier et al. 2003; Colobert et al. 2006; Betker et al. 2006) which is a global outcome measure and does not fully describe segmental postural behaviors. A perturbed postural system does not necessarily respond only at the driving frequency (Creath et al. 2005; Loughlin et al. 1996; Loughlin and Redfern 2001) and one of the advantages of this statistical model is that it is not limited to describing behavior just at the driving frequency. We believe that this model will enable us to explore the full bandwidth of adaptive postural control during complex tasks.

Acknowledgements: This work was supported by National Institute of Health grants DC01125 and DC05235 from the NIDCD and AG16359. We gratefully acknowledge VRCO for supplying CAVE library software and the Ascension Tracker Daemon.

\section{REFERENCES}

Baker, G.L., Gollub, J.P. (1990). Chaotic Dynamics, pp 134135. $1^{\text {st }}$ Ed. Cambridge University Press, Cambridge.

Barbier, F., Allard, P., Guelton, K., Colobert, B., GodillonMaquinghen, A.P. (2003). Estimation of the 3D center of mass excursion from force plate data during standing. IEEE Transactions on Neural Systems \& Rehabilitation Engineering, 11 (1), 31-37.Bendat, J.S., Piersol, A.G. (1993). Engineering applications of correlation and spectral analysis, pp 45-46. 2nd Ed. John Wiley and Sons, Inc. New York, NY.

Betker, A.L., Moussavi, Z.M.K., Szturm, T. (2006). Center of mass approximation and prediction as a function of body acceleration. IEEE Transactions on Biomedical Engineering, 53 (4), 686-693.

Bottaro, A., Casadio, M., Morasso, P.G., Sanguineti, V. (2005). Body sway during quiet standing: Is it the residual chattering of an intermittent stabilization process? Human Movement Science, 24, 588-615.
Buchanan, J.J., Horak, F.B. (2003). Voluntary control of postural equilibrium patterns. Behavioral Brain Research, 143, 121-140.

Buchanan, J.J., Horak, F.B. (2001). Transitions in a postural task: do the recruitment and suppression of degrees of freedom stabilize posture? Exp Brain Res, 139, 482-494.

Carroll, J.P., Freedman, W. (1993). Nonstationary properties of postural sway. J Biomech, 26, 409-416.

Collins, J.J., De Luca, C.J. (1993). Open-loop and closedloop control of posture: a random-walk analysis of center-ofpressure trajectories. Exp Brain Res, 95, 308-318.

Colobert, B., Crétual, B., Allard, P., Delamarche, P. (2006). Force-plate based computation of ankle and hip strategies from double-inverted pendulum model. Clinical Biomechanics, 21 (4), 427-434.

Creath, R., Kiemel, T., Horak, F., Peterka, R., Jeka, J. (2005). A unified view of quiet and perturbed stance: simultaneous co-existing excitable modes. Neurosci Lett, 377, 75-80.

Guelton, K., Delprat, S., Guerra, T.M. (2008). An alternative to inverse dynamics joint torques estimation in human stance based on a Takagi-Sugeno unknown inputs observer in the descriptor form. Control Engineering Practice, 16 (12), 1414-1426.

Gurses, S., Dhaher, Y., Hain, T.C., Keshner, E.A. (2005). Perturbation parameters associated with nonlinear responses of the head at small amplitudes. Chaos, 15, 23905.

Horak, F.B. (2006). Mechanistic and physiological aspects "Postural orientation and equilibrium: what do we need to know about neural control of balance to prevent falls?" Age and Ageing, 35-S2, ii7-iil1.

Jonsson, E., Seiger, A., Hirschfeld, H. (2005). Postural steadiness and weight distribution during tandem stance in healthy young and elderly adults. Clin Biomech (Bristol, Avon), 20, 202-208.

Jonsson, E., Seiger, A., Hirschfeld, H. (2004). One-leg stance in healthy young and elderly adults: a measure of postural steadiness? Clin Biomech (Bristol, Avon), 19, 688-694.

Keshner, E.A., Kenyon, R.V., Langston, J. (2004). Postural responses exhibit multisensory dependencies with discordant visual and support surface motion. J Vest ResearchEquilibrium \& Orientation, 14 (4), 307-319.

Keshner, E.A. (2003). Head-trunk coordination during linear anterior-posterior translations. J Neurophysiol, 89, 18911901.

Keshner, E.A., Kenyon, R.V. (2000). The influence of an immersive virtual environment on the segmental organization of postural stabilizing responses. $J$ Vest ResearchEquilibrium \& Orientation, 10 (4-5), 207-219.

Keshner, E.A., Cromwell, R.L., Peterson, B.W. (1995). Mechanisms controlling human head stabilization. II. Headneck characteristics during random rotations in the vertical plane. J Neurophysiol, 73, 2302-2312. 
Kuo, A.D., Speers, R.A., Peterka, R.J., Horak, F.B. (1998). Effect of altered sensory conditions on multivariate descriptors of human postural sway. Exp Brain Res, 122, 185-195.

Loughlin, P.J., Redfern, M.S., Furman, J.M. (1996). Timevarying characteristics of visually induced postural sway. IEEE Trans Rehabil Eng, 4, 416-424.

Nashner, L.M. (1982). Adaptation of human movement to altered environments. Trends Neurosci, 5, 358-361.

Rankin, J.K., Woollacott, M.H., Shumway-Cook, A., Brown, L.A. (2000). Cognitive influence on postural stability: a neuromuscular analysis in young and older adults. $J$ Gerontol A Biol Sci Med Sci, 55, M112-119.

Redfern, M.S., Jennings, R.J., Martin, C., Furman, J.M. (2001). Attention influences sensory integration for postural control in older adults. Gait \& Posture, 14, 211-216.

Riley, M.A., Clark, S. (2003). Recurrence analysis of human postural sway during the sensory organization test. Neurosci Lett, 342, 45-48.

Schumann, T., Redfern, M.S., Furman, J.M., El-Jaroudi, A., Chaparro, L.F. (1995). Time-frequency analysis of postural sway. J Biomech, 28, 603-607.

Shumway-Cook, A., Woolacott, M. (1999). Attentional demands and postural control:the effect of sensory context. $J$ Gerontol, 55A, M10-16.

Speers, R.A., Kuo, A.D., Horak, F.B. (2002). Contributions of altered sensation and feedback responses to changes in coordination of postural control due to aging. Gait and Posture, 16, 20-30.

Woollacott, M., Shumway-Cook, A. (2002). Attention and the control of posture and gait: a review of an emerging area of research. Gait \& Posture, 16, 1-14. 